\title{
TIME-DEPENDENT INVARIANTS FOR DIRAC EQUATION AND NEWTON-WIGNER POSITION OPERATOR
}

\author{
V.I. Man'koø and R.V. Mendes \\ Grupo de Física-Matemática, Complexo II, Universidade de Lisboa \\ Av. Prof. Gama Pinto, 2, 1699 Lisboa Codex, Portugal
}

\begin{abstract}
For Dirac equation, operator-invariants containing explicit time-dependence in parallel to known time-dependent invariants of nonrelativistic Schrödinger equation are introduced and discussed. As an example, a free Dirac particle is considered and new invariants are constructed for it. The integral of motion, which is initial Newton-Wigner position operator, is obtained explicitly for a free Dirac particle. For such particle with kick modeled by delta-function of time, the time-depending integral, which has physical meaning of initial momentum, is found.
\end{abstract}

\section{Introduction}

The usual time-independent integrals of motion of stationary quantum system like energy or angular momentum are related to symmetry of the Hamiltonian, which is connected with time translational symmetry or rotational symmetry of the potential [1]. For nonrelativistic quantum

\footnotetext{
${ }^{1}$ on leave from Lebedev Physical Institute, Moscow, Russia
} 
systems with nonstationary Hamiltonians (for example, for the parametric oscillator), quadratic in position and momentum integrals of motion of classical problem, known long time ago [2], were shown to exist for quantum problem as well [3]. A connection of finding wave function of nonstationary Hamiltonians with existence of time-dependent integrals of motion has been also elaborated [4.

For the same problem of nonrelativistic parametric oscillator, linear in position and momentum time-dependent integrals of motion have been found [5-7] and have been related to the Green function of Schrödinger equation [8]. A connection of the integrals of motion with quantum propagator turns out to be general property related to Schwinger action principle [9]. Futhermore, an extension of the Nöther theorem from time-independent integrals of motion to time-dependent integrals of motion has been done [10-13]. The method of finding time-dependent invariants was developed in recent works [14-17].

The time-independent integrals of motion as energy and angular momentum are used as well in relativistic problems of scalar and spinor particles in the framework of Klein-Gordon or Dirac equations [1]. But up to our knowledge till now the time-dependent integrals of motion for relativistic quantum systems have not been discussed and there is still no one example of timedependent integral of motion for relativistic quantum system.

The aim of this work is to find new time-dependent integrals of motion for free Dirac particle and to extend the results known for nonrelativistic propagator of Schrödinger equation, which may be obtained as eigenfunction of time-dependent invariants [8] to the case of Dirac equation.

The problem of position operator in relatvistic domain is more complicated than for Schrödinger wave mechanics. There are known results for Dirac equation to introduce an operator playing role 
of position operator [18, 19]. Newton-Wigner position operator is intensively discussed, in recent publications as well 20]. Thus, another goal of our work is to construct a time-dependent integral of motion, which coincides with Newton-Wigner position operator for initial time moment. Production of electron-positron pairs in heavy ion collisions [21] was studied recently in frame of the model of short pulses described by delta-kickes [22]. Dirac equation was used in which a potential was switched in for very short time corresponding to ultrafast interaction of high energy of colliding ions. In this connection, it is worthy to investigate what type of integrals of motion does exist in such potential. We construct explicitly expressions for some time-dependent integrals of motion for Dirac equation with short kickes modeled by delta-function of time.

The paper is organized as follows. In Section 2, we review the known time-dependent invariants for free nonrelativistic scalar Schrödinger particle (readers having interest to detail review we address to Refs. [23, 24] ) and generalize the nonrelativistic invariants to the case of free Dirac particle. In Section 3, we discuss the time-dependent invariants and their properties for Dirac particle in electromagnetic field. In Section 4, we discuss Newton-Wigner position operator and its time-dependent analog, which is an integral of motion for Dirac free particle. In Section 5, an extension of the integrals of motion found for free Dirac particle is done for the kicked Dirac particle in frame of the delta-pulse model.

\section{Invariants for Free Dirac Particle}

Let us consider a free Dirac equation in standard form

$$
i \frac{\partial \psi}{\partial t}=(\alpha \hat{p}+\beta m) \psi ; \quad \hbar=1=c
$$


where $p_{i}=-i \partial / \partial x_{i} \quad(i=1,2,3)$ are the components of 3-vector $\hat{p}, m$ is constant mass, and the $4 \times 4-$ matrices $\alpha_{i}$ and $\beta$ have the form

$$
\alpha=\left(\begin{array}{cc}
0 & \sigma \\
\sigma & 0
\end{array}\right) ; \quad \beta=\left(\begin{array}{cc}
1 & 0 \\
0 & -1
\end{array}\right)
$$

where the vector-matrix $\alpha$ has three components $\alpha_{i}$. The Pauli $2 \times 2-$ matrices which are components of vector-matrix $\sigma$ are chosen to be

$$
\sigma_{1}=\left(\begin{array}{cc}
0 & 1 \\
1 & 0
\end{array}\right) ; \quad \sigma_{2}=\left(\begin{array}{cc}
0 & -i \\
i & 0
\end{array}\right) ; \quad \sigma_{3}=\left(\begin{array}{cc}
1 & 0 \\
0 & -1
\end{array}\right)
$$

The aim of this section is to discuss for a free Dirac particle the existence of a time-dependent integral of the motion.

Let us remind that for a free Schrödinger particle with the Hamiltonian

$$
H=\frac{\hat{p}^{2}}{2 m} ; \quad p_{i}=-i \frac{\partial}{\partial x_{i}} ; \quad i=1,2,3
$$

there exist the following invariants: momentum operator described by 3 -vector

$$
\hat{p}_{0}(t)=\hat{p}
$$

which commutes with the Hamiltonian (4), and the initial coordinate vector-operator $\hat{x}_{0}(t)$ with the components

$$
x_{0 i}(t)=x_{i}+\frac{i t}{m} \frac{\partial}{\partial x_{i}} .
$$

Here $x_{i}$ are components of 3 -vector $\hat{x}$. The vector-operators $\hat{x}_{0}(t)$ and $\hat{p}_{0}(t)$ have the property that

$$
\left\langle\psi(t)\left|\hat{x}_{0}(t)\right| \psi(t)\right\rangle=\langle\psi(0)|\hat{x}| \psi(0)\rangle
$$

and

$$
\left\langle\psi(t)\left|\hat{p}_{0}(t)\right| \psi(t)\right\rangle=\langle\psi(0)|\hat{p}| \psi(0)\rangle
$$


for any solution to the Schrödinger equation. At the same time, these operators may be expressed in terms of the evolution operator $\hat{U}(t)$ of the free Schrödinger particle. This evolution operator satisfies the equation

$$
i \frac{\partial \hat{U}}{\partial t}=\frac{\hat{p}^{2}}{2 m} \hat{U}
$$

with the initial conditions

$$
\hat{U}(0)=\hat{E}
$$

The operator $\hat{E}$ is the identity operator. In terms of the operator $\hat{U}(t)$, the invariant $\hat{x}_{0}(t)($ (6) $)$ is expressed as follows

$$
\hat{x}_{0}(t)=\hat{U}(t) \hat{x} \hat{U}^{-1}(t)
$$

and the operator $\hat{p}_{0}(t)$ (5) is expressed in the similar manner

$$
\hat{p}_{0}(t)=\hat{U}(t) \hat{p} \hat{U}^{-1}(t)
$$

The operator $\hat{U}(t)$ is the solution to Eq. (9) and has the form

$$
\hat{U}(t)=\exp \left(-i t \frac{\hat{p}^{2}}{2 m}\right)
$$

Thus, the exact forms of the operators $\hat{x}_{0}$ and $\hat{p}_{0}$ are the result of commuting of the operator $\hat{U}$ (12) either with operator $\hat{p}$ or with operator $\hat{x}$ in formulas (11) and (10), correspondingly. At the same time, these operators satisfy the system of equations

$$
\begin{aligned}
& \frac{\partial \hat{x}_{0}}{\partial t}+i\left[H, \hat{x}_{0}\right]=0 \\
& \frac{\partial \hat{p}_{0}}{\partial t}+i\left[H, \hat{p}_{0}\right]=0
\end{aligned}
$$

For this simple problem, we can use a trick to guess the form of the operators (5) and (6) on the basis of classical intuition. 
Let us write down the classical solution for the free motion equation. We have

$$
\begin{aligned}
p(t) & =p_{0} ; \\
x(t) & =x_{0}+v_{0} t ; \\
p & =m v .
\end{aligned}
$$

Here $p_{0}$ and $v_{0}\left(p_{0}\right)$ are the initial values of the coordinate and velocity (momentum) of the particle. Expressing these initial values in terms of the instant values $p(t)$ and $x(t)$, in view of (15), we have

$$
\begin{aligned}
& p_{0}=p(t) \\
& x_{0}=x-t \frac{p(t)}{m} .
\end{aligned}
$$

"Quantizing" these initial values we obtain the quantum invariants (5) and (6).

From formulas (10) and (11), it follows that the propagator of a free Schrödinger particle $G\left(x, x^{\prime}, t\right)$ satisfies the system of equations

$$
\begin{aligned}
& \hat{x}_{0}(t) G\left(x, x^{\prime}, t\right)=x^{\prime} G\left(x, x^{\prime}, t\right) ; \\
& \hat{p}_{0}(t) G\left(x, x^{\prime}, t\right)=i \frac{\partial}{\partial x^{\prime}} G\left(x, x^{\prime}, t\right) .
\end{aligned}
$$

Thus, knowing invariants (5) and (6) we have the equations for matrix elements of evolution operator in the coordinate representation.

Now let us discuss what properties of the Schrödinger invariants $\hat{p}_{0}$ and $\hat{x}_{0}$ may be generalized for the Dirac particle. It is the general problem. But first we have to consider the problem applying it to the simplest case of a free particle. Equation (1) is the equation of the same evolutionary type as the Schrödinger equation. Thus, the $4 \times 4-$ matrix $U_{D}(t)$ may be introduced as evolution 
operator. This operator-matrix satisfies the equation

$$
i \frac{\partial U_{D}}{\partial t}=H_{D} U_{D}
$$

where the Hamiltonian $H_{D}$ is the $4 \times 4$-operator-matrix

$$
H_{D}=\alpha \hat{p}+\beta m
$$

The solution to (20) has the same form that in the Schrödinger case

$$
U_{D}(t)=\exp \left(-i H_{D} t\right)
$$

Let us now generalize formulas (10) and (11) of the Schrödinger case to the Dirac case. We have the operators

$$
\begin{aligned}
& p_{0 D}(t)=\exp \left(-i H_{D} t\right) \hat{p} \exp \left(i H_{D} t\right) \\
& x_{0 D}(t)=\exp \left(-i H_{D} t\right) \hat{x} \exp \left(i H_{D} t\right) .
\end{aligned}
$$

Equation (23) immediately gives the equality for $p_{0 D}(t)$, namely,

$$
p_{0 D}(t)=\hat{p}
$$

as well as it was in the Schrödinger case, since the Hamiltonian (21) of the Dirac free particle commutes with the momentum $\hat{p}$. In order to calculate expression (24), we could use the CampbellHausdorf formula

$$
e^{B} A e^{-B}=A+[B, A]+\frac{1}{2 !}[B,[B, A]]+\cdots+\frac{1}{n !}[B,[B[\ldots[B, A]]] \ldots]+\cdots
$$

But since the first commutator (if one takes $B=-i t H_{D}$ and $A=x_{i}$ which is the $\mathrm{i}$-th component of the vector $\hat{x}$ )

$$
[B, A]=\left[-i t\left(\alpha_{k} p_{k}+\beta m\right), x_{i}\right]=-i t\left(-i \delta_{i k}\right) \alpha_{k}=-t \alpha_{i}
$$


is not $c$-number, we need to calculate the next commutators in (26)

$$
[B,[B, A]]=\left[-i t\left(\alpha_{k} p_{k}+\beta m\right),-t \alpha_{i}\right]=i t^{2}\left(\left[\alpha_{k} \alpha_{i}\right] p_{k}+m\left[\beta \alpha_{i}\right]\right)
$$

The matrices $\alpha_{i}$ and $\beta$ obey the known algebra

$$
\begin{aligned}
\alpha_{i} \alpha_{k}+\alpha_{k} \alpha_{i} & =2 \delta_{i k} ; \\
\alpha_{i} \beta+\beta \alpha_{i} & =0 ; \\
\alpha_{i}^{2}=\beta_{i}^{2} & =1 .
\end{aligned}
$$

From this algebra, taking into account (28) it is not obvious how to use these formulas for calculating each term in series (26) as well as the total sum.

To evaluate this sum, we use the specific property of the Dirac Hamiltonian for free particle. Let us introduce $-i t=\tau$. Then the matrix $H_{D}$ has the form

$$
H_{D}=\left(\begin{array}{cc}
m & \sigma \hat{p} \\
\sigma \hat{p} & -m
\end{array}\right) \text {. }
$$

The square of this matrix is the following diagonal matrix

$$
H_{D}^{2}=\left(\begin{array}{cc}
m^{2}+\hat{p}^{2} & 0 \\
0 & m^{2}+\hat{p}^{2}
\end{array}\right)=\varepsilon^{2} E
$$

where

$$
\varepsilon^{2}=\hat{p}^{2}+m^{2}
$$

and $E$ is the $4 \times 4$-identity matrix. Then one has

$$
H_{D}^{3}=\varepsilon^{2} H_{D}, \quad H_{D}^{4}=\varepsilon^{4} E, \ldots, H_{D}^{2 n-1}=\varepsilon^{2 n-1} \frac{H_{D}}{\varepsilon}, \quad H_{D}^{2 n}=\varepsilon^{2 n} E
$$


Thus,

$$
e^{\tau H_{D}}=E+\tau \varepsilon H_{D}+\frac{\varepsilon^{2} \tau^{2}}{2 !} E+\frac{\varepsilon^{3} \tau^{3}}{3 !} \frac{H_{D}}{\varepsilon}+\cdots=E \cosh \varepsilon \tau+\frac{H_{D}}{\varepsilon} \sinh \varepsilon \tau
$$

This operator in the matrix form is

$$
e^{\tau H_{D}}=\left(\begin{array}{cc}
\cosh \varepsilon \tau+\varepsilon^{-1} m \sinh \varepsilon \tau & \varepsilon^{-1} \sigma \hat{p} \sinh \varepsilon \tau \\
\varepsilon^{-1} \sigma \hat{p} \sinh \varepsilon \tau & \cosh \varepsilon \tau-\varepsilon^{-1} m \sinh \epsilon \tau
\end{array}\right)
$$

The matrix $\varepsilon^{-\tau H_{D}}$ differs only by sign before the term with sinus hyperbolic. At $\tau=0$, this matrix coincides with the identity matrix $E$.

The Green function $G_{i k}\left(x, x^{\prime}, t\right)$ is the $4 \times 4$-matrix $(i, k=1,2,3,4)$ containing matrix elements of the evolution operator $U_{D}(t)$. It is connected by the relation similar to (18) and (19) with constructed invariants $x_{0 D}(t)$ and $p_{0 D}(t)=\hat{p}$ which are $4 \times 4$-matrices as well. So

$$
p_{0 D}(t) G_{i k}\left(x, x^{\prime}, t\right)=i \frac{\partial}{\partial x^{\prime}} G_{i k}\left(x, x^{\prime}, t\right)
$$

and

$$
\left(x_{0 D}(t)\right)_{i i^{\prime}} G_{i^{\prime} k}\left(x, x^{\prime}, t\right)=x_{i}^{\prime} G_{i k}\left(x, x^{\prime}, t\right)
$$

Here we have in mind summation over two equal indices $i^{\prime}$ from 1 to 4 and no summation over index $i$. The operators in the matrices $x_{0 D}$ and $p_{0 D}$ act on the first argument $x$ of the propagator $G_{i k}\left(x, x^{\prime}, t\right)$

Now we will write down four matrix elements of the matrix $x_{0 D}(t)$. This matrix consists of four $2 \times 2$-blocks. We will label these blocks as $\left(x_{0}\right)_{11},\left(x_{0}\right)_{12},\left(x_{0}\right)_{21}$, and $\left(x_{0}\right)_{22}$. (In the case of the free Dirac particle, the matrix structure of the operator $p_{0 D}(t)$ is simple because it is proportianal to unity $4 \times 4$-matrix.) Thus, the $2 \times 2$-block $\left(x_{0}(t)\right)_{11}$ of invariant $x_{0 D}$ is the following one

$$
\left(x_{0}(t)\right)_{11}=\cosh \varepsilon \tau \hat{x} \cosh \varepsilon \tau-\frac{m^{2}}{\varepsilon} \sinh \varepsilon \tau \hat{x} \frac{1}{\varepsilon} \sinh \varepsilon \tau
$$




$$
\begin{aligned}
& +m\left[\frac{1}{\varepsilon} \sinh \varepsilon \tau \hat{x} \cosh \varepsilon \tau-\cosh \varepsilon \tau \hat{x} \frac{1}{\varepsilon} \sinh \varepsilon \tau\right] \\
& -\frac{\sigma \hat{p}}{\varepsilon} \sinh \varepsilon \tau \hat{x} \frac{1}{\varepsilon} \sinh \varepsilon \tau .
\end{aligned}
$$

Here

$$
\varepsilon=\sqrt{m^{2}+\hat{p}^{2}} ; \quad p_{i}=-i \frac{\partial}{\partial x_{i}}(i=1,2,3) ; \quad \tau=-i t
$$

We must take into account the order of the operators in the product in this formula because they do not commute. The next block $\left(x_{0}(t)\right)_{12}$ has the form

$$
\begin{aligned}
\left(x_{0}(t)\right)_{12} & =m\left[\frac{\sigma \hat{p}}{\varepsilon} \sinh \varepsilon \tau \hat{x} \frac{1}{\varepsilon} \sinh \varepsilon \tau-\frac{1}{\varepsilon} \sinh \varepsilon \tau \hat{x} \frac{\sigma \hat{p}}{\varepsilon} \sinh \varepsilon \tau\right] \\
& +\frac{\sigma \hat{p}}{\varepsilon} \sinh \varepsilon \tau \hat{x} \cosh \varepsilon \tau-\cosh \varepsilon \tau \hat{x} \frac{\sigma \hat{p}}{\varepsilon} \sinh \varepsilon \tau
\end{aligned}
$$

Of course, we can use the commutator $\left[x_{i}, p_{k}\right]=i \delta_{i k},(i, k=1,2,3)$ in order to rewrite the terms in these formulas. For the block $\left(x_{0}\right)_{21}$, we have the formula

$$
\begin{aligned}
\left(x_{0}(t)\right)_{21} & =m\left[\frac{1}{\varepsilon} \sinh \varepsilon \tau \hat{x} \frac{\sigma \hat{p}}{\varepsilon} \sinh \varepsilon \tau\right]-\frac{\sigma \hat{p}}{\varepsilon} \sinh \varepsilon \tau \hat{x} \frac{1}{\varepsilon} \sinh \varepsilon \tau \\
& +\frac{\sigma \hat{p}}{\varepsilon} \sinh \varepsilon \tau \hat{x} \cosh \varepsilon \tau-\cosh \varepsilon \tau \hat{x} \frac{\sigma \hat{p}}{\varepsilon} \sinh \varepsilon \tau .
\end{aligned}
$$

The block $\left(x_{0}\right)_{22}$ of the $4 \times 4$-matrix-invariant $x_{0}(t)$ has the form

$$
\begin{aligned}
\left(x_{0}(t)\right)_{22} & =\cosh \varepsilon \tau \hat{x} \cosh \varepsilon \tau-\frac{m^{2}}{\varepsilon^{2}} \sinh \varepsilon \tau \hat{x} \sinh \varepsilon \tau \\
& +m\left[\frac{1}{\varepsilon} \cosh \varepsilon \tau \hat{x} \sinh \varepsilon \tau-\frac{1}{\varepsilon} \sinh \varepsilon \tau \hat{x} \cosh \varepsilon \tau\right] \\
& -\frac{\sigma \hat{p}}{\varepsilon} \sinh \varepsilon \tau \hat{x} \frac{\sigma \hat{p}}{\varepsilon} \frac{\sinh \varepsilon \tau}{\varepsilon} .
\end{aligned}
$$

From the structure of these blocks, it is evident that it is not easy to obtain the invariant-operator $\hat{x}_{0}$ using the Campbell-Hausdorf formula. Thus, we have generalized the integrals of the motion 
for the free Schrödinger particle to the case of the free Dirac particle. By constructing the $4 \times 4-$ matrix-operator $\hat{p}_{0 D}$ and $\hat{x}_{0 D}$ obey the Weyl-Heisenberg algebra with commutation relations

$$
\left[p_{0 D k}, x_{0 D l}\right]=-i \delta_{l k}
$$

From these operators, it is possible to construct some invariants with different commutation relations. So taking the operator

$$
L_{i}(t)=\varepsilon_{i l k} x_{0 D l} p_{0 D k}, \quad i, k, l=1,2,3,
$$

one can check that the three time-dependent operators obey the commutation relations of the angular momentum

$$
\left[L_{i}(t), L_{k}(t)\right]=i \varepsilon_{i k l} L_{l}(t)
$$

On the other hand, at $t=0$ these operators coincide with the orbital angular momentum operator $L(t=0)=[\hat{x} \times \hat{p}]$, which does not conserve, but the operator (44) is invariant. Along with the operators $p_{0 D}$, the invariants $L(t)$ form the Lie algebra of the generators of the motion group in three-dimensional space. For the free Dirac particle, the conserved operator (which is the sum of orbital and spin momenta) $j=[\hat{x} \times \hat{p}]+\sigma / 2$. It commutes with the Dirac energy operator $H_{D}$. Due to this, one can write down the equality

$$
U_{D} L(t=0) U_{D}^{-1}+\frac{1}{2} U_{D} \sigma U_{D}^{-1}=L+\frac{\sigma}{2}
$$

Thus, the orbital angular momentum described by the operator $L(t)$ may be expressed as

$$
L(t)=L(t=0)+\frac{\sigma}{2}-\frac{1}{2} U_{D} \sigma U_{D}^{-1}
$$

The matrix $U_{D}(t)[\sigma / 2] U_{D}^{-1}(t)=S(t)$ is the following integral of the motion which describes the initial spin operator. It may be obtained in the form of $4 \times 4$-matrix operator using formulas (24) 
and (38)-(42) by the simple substitution: $\hat{x} \rightarrow \sigma$ in all these formulas, all other terms in the formulas being unchanged.

\section{General Consideration of Operator-Invariants for Dirac}

\section{Particle}

The analysis of the previous section was based on the fact that the free Dirac particle motion may be described by the equation of an evolutianary type with the concrete Hamiltonians (20)(21). After that, we used the free propagator or evolution operator for free Dirac particles in order to construct the invariants $p_{0 D}(t), x_{0 D}(t)$, and $L(t)$ which contained dependence on time. But it is clear that the same procedure may be fulfilled not only for the free Dirac particle. Any evolutianary type equation for bispinor $\psi$ of the kind

$$
i \frac{\partial \psi}{\partial t}=H_{D} \psi
$$

with the Hamiltonian

$$
H_{D}=\left\{\alpha\left[\hat{p}-\frac{e}{c} A(x, t)\right]+\beta m+V(x, t)\right\}
$$

in which we have taken into account also the possibility of presence of electric and magnetic fields,

posesses the evolution operator or propagator $\hat{U}_{D}(t)$, which makes the bispinor $\psi(t)$ from the initial bispinor $\psi(0)$ satisfying Eq. (48)

$$
\psi(t)=U_{D}(t) \psi(0)
$$

If for the more complicated cases with electric and magnetic fields the evolution operator $\hat{U}_{D}(t)$ 
is found, we can construct invariants $\hat{I}_{D}(t)$ by the formula

$$
I_{D}(t)=U_{D}(t) I_{D}(0) U_{D}^{-1}(t)
$$

The properties of the operator $I_{D}(t)$ are very similar to the properties of corresponding integrals of the motion in the case of Schrödinger equation and, of course, these Dirac invariants-operators tend to the integrals of the motion of the Schrödinger particle in nonrelativistic limit. Some theorems about Schrödinger integrals of the motion may be transfered to the case of the Dirac equation. For example, a function of the Dirac invariant-operator is another Dirac invariant-operator. This statement means, that if we have a function $f\left(I_{D}(t)\right)$, this function may be always represented in the form

$$
f\left(I_{D}(t)\right)=U_{D}(t) f\left(I_{D}(0)\right) U_{D}^{-1}(t)
$$

But this formula follows from the obvious property

$$
f\left(U_{D}(t) I_{D}(0) U_{D}^{-1}(t)\right)=U_{D}(t) f\left(I_{D}(0)\right) U_{D}^{-1}(t)
$$

For example, for any power series with respect to the operator $I_{D}(t)$ it can be checked and extended for more complicated function. From this property, it follows that a free Dirac particle has also the following invariants:

$$
p_{0 D}^{2}, \quad x_{0 D}^{2}, \quad\left(p_{0 D} x_{0 D}\right), \quad p_{0 D} L(t)
$$

The eigenvalues of the Dirac invariants-operators do not depend on time. It means that if we search for the solution to the equation

$$
I_{D}(t) \psi(t)=\lambda(t) \psi(t)
$$

where bispinor $\psi(t)$ is a solution to the Dirac equation, the function $\lambda(t)$ is constant and does not 
depend on time. This statement follows from simple checking. We have

$$
U_{D}(t) I_{D}(0) U_{D}^{-1}(t) U_{D}(t) \psi(0)=\lambda(t) U_{D}(t) \psi(0)
$$

After simple algebra, it is obvious that $\lambda(t)$ is eigenvalue of the time-independent operator $I_{D}(0)$ and due to this it does not depend on time as well.

The third statement which may be generalized from the Schrödinger example is that if we have a solution to the Dirac equation $\psi(t)$ and act on it by the Dirac invariant operator $I_{D}(t)$, the new bispinor $\varphi(t)$ such that

$$
\varphi(t)=I_{D}(t) \psi(t)
$$

turns out to be the solution to the Dirac equation, too. This statement may be proved by simple substitution. For the solution $\psi(t)$, we have the expression

$$
\psi(t)=U_{D}(t) \psi(0)
$$

Then, we have

$$
I_{D}(t) \psi(t)=U_{D}(t) I_{D}(0) U_{D}^{-1}(t) U_{D}(t) \psi(0)
$$

but the bispinor

$$
\varphi(t)=U_{D}(t) \varphi(0)
$$

where

$$
\varphi(0)=I_{D}(0) \psi(0)
$$

satisfies the Dirac equation due to the property of the Dirac propagator $U_{D}(t)$.

The matrix elements of the Dirac evolution operator $U_{D}(t)$ in any representation satisfy the relations analogous to (36) and (37) for the free Dirac particle. In the coordinate representation, for any Dirac Hamiltonian if we found the operators $\hat{x}_{0}(t)$ and $\hat{p}_{0}(t)$, these equations hold. 


\section{Newton-Wigner Operator}

The integral of motion, which is an initial position operator, turns out to play important role in considering a nonrelativistic quantum system [23, 24]. Due to this for relativistic Dirac particle it is worthy to study an analogous integral of motion, which plays the role of initial position of the particle. For the Dirac particle, the position operator itself has some pecularities and it differs from the position operator of nonrelativistic particle. The most appropriate operator playing the role of the position operator of the Dirac particle was considered by Newton and Wigner [18, [19].

We will construct the time-dependent integral of motion, which has the physical meaning of the initial position operator. It means that this integral of motion at the initial moment of time must coincide with the Newton-Wigner position operator.

The Newton-Wigner position operator [18, 19 has the form (see, for example, 20])

$$
Q_{k}=\Lambda_{+}(\hat{p})\left(1+\gamma_{0}\right) \sqrt{\frac{p_{0}}{p_{0}+m}}\left(i \frac{\partial}{\partial p_{k}}\right) \sqrt{\frac{p_{0}}{p_{0}+m}} \Lambda_{+}(\hat{p}),
$$

where

$$
p_{0}^{2}=p_{1}^{2}+p_{2}^{2}+p_{3}^{2}
$$

and the projector

$$
\Lambda_{+}(\hat{p})=\frac{1}{2}\left(1+\frac{\alpha \hat{p}+\beta m}{p_{0}}\right) .
$$

Also we used for the operator of coordinate in momentum representation the notation

$$
\hat{x}_{i}=i \frac{\partial}{\partial p_{k}}, \quad i=1,2,3
$$

For free Dirac particle, one can construct 3-vector-operator $Q_{k}(t)$, which is integral of motion, using the general construction and invariant found in previous section, i.e.,

$$
Q_{k}(t)=U_{D}(t) Q_{k} U_{D}^{-1}(t)
$$


Since the operators

$$
U_{D}(t) p_{0} U_{D}^{-1}(t)=p_{0}
$$

and

$$
U_{D}(t) \Lambda_{+}(\hat{p}) U_{D}^{-1}(t)=\Lambda_{+}(\hat{p})
$$

are integrals of motion for free particle, the integral of motion, which for initial time $t=0$ coincides with the Newton-Wigner position operator is

$$
Q_{k}(t)=\Lambda_{+}(\hat{p})\left(1+\gamma_{0}(t)\right) \sqrt{\frac{p_{0}+m}{p_{0}}} x_{k}(t) \sqrt{\frac{p_{0}+m}{p_{0}}} \Lambda_{+}(\hat{p})
$$

Here the integral of motion $x_{k}(t) \equiv x_{0 D k}(t)$ is given in coordinate representation by (38)-(42) and in momentum representation by the same formulas (38)-(42), in which the operator $\hat{x}$ is replaced by $i \partial / \partial \hat{p}$. The integral of motion

$$
\gamma_{0}(t)=U_{D}(t) \gamma_{0} U_{D}^{-1}(t)
$$

in explicit form is

$$
\gamma_{0}(t)=\left(\begin{array}{cc}
\cosh ^{2} \varepsilon \tau-\varepsilon^{2}\left(m^{2}-\hat{p}^{2}\right) \sinh ^{2} \varepsilon \tau & -2 \varepsilon^{-1} \sigma \hat{p} \sinh \varepsilon \tau\left(\varepsilon^{-1} \sinh \varepsilon \tau+\cosh \varepsilon \tau\right) \\
-2 \varepsilon^{-1} \sigma \hat{p} \sinh \varepsilon \tau\left(\varepsilon^{-1} \sinh \varepsilon \tau-\cosh \varepsilon \tau\right) & \varepsilon^{-2}\left(m^{2}-\hat{p}^{2}\right) \sinh ^{2} \varepsilon \tau-\cosh ^{2} \varepsilon \tau
\end{array}\right)
$$

Thus, we get 3 -vector-operator $\widehat{Q}_{k}(t)$, which is the integral of motion of free Dirac particle. This integral of motion "remembers" its initial maximally localized position in space.

\section{Short Pulses for Dirac Partilce}

Let us consider the model, in which the interaction term for Dirac particle is the term $V(x, t)$ 
in (49), i.e., we have

$$
H_{D}=\alpha \hat{p}+\beta m+V(x, t) .
$$

We study what influence produces very short pulse of the potential. It is motivated by discussion [22] of heavy ion collisions so fast passing each the other in process of high energy reaction that the perturbation of the potential field lasts very short time in comparison with the periods of all motions in the system. In this case, the model of the potential $V(x, t)$ may be described by $\delta$-function of time

$$
V(x, t)=\kappa V(x) \delta\left(t-t^{\prime}\right)
$$

We study explicitly how the propagator for the free Dirac particle is changed if the potential of the type (71) switches in. This problem is interesting not only in the context of studying pair production in the process of charge collisions but also as the simple exactly solvable model of relativistic particle with nonstationary Dirac Hamiltonian. Then solving Eq. (48) with the Hamiltonian (70), (71) we can find that the propagator of the particle with $\delta$-type interaction in time may be factorized

$$
U_{D}\left(t_{\mathrm{f}}, t_{\text {in }}\right)=U_{\mathrm{f}}\left(t_{\mathrm{f}}, t^{\prime}\right) \exp (-i \kappa V) U_{\mathrm{f}}\left(t^{\prime}, t_{\text {in }}\right)
$$

Here $U_{\mathrm{f}}\left(t_{2}, t_{1}\right)$ is the propagator of free particle and the influence of $\delta$-pulse at the moment $t^{\prime}$ gives the factor $\exp (-i \kappa V)$. The free propagator has been calculated in previous sections and is described by formula (35). If we introduce the notation

$$
-i\left(t-t^{\prime}\right)=\tau_{1} ; \quad-i\left(t^{\prime}-t_{0}\right)=\tau_{2} ; \quad \varepsilon_{1}=\sqrt{p_{1}^{2}+m^{2}} ; \quad \varepsilon_{2}=\sqrt{p_{2}^{2}+m^{2}}
$$

and

$$
(\exp [-i \kappa V])_{p_{1} p_{2}}=\int \exp \left[i\left(p_{1}-p_{2}\right) x-i \kappa V(x)\right] d x
$$


in momentum representation, the propagator of the particle $G\left(p_{1}, p_{2}, t, t_{0}\right)$ with $\delta$-pulse takes the form

$$
G\left(p_{1}, p_{2}, t, t_{0}\right)=(\exp [-i \kappa V])_{p_{1} p_{2}} \prod_{i=1}^{2}\left(\begin{array}{cc}
\cosh \varepsilon_{i} \tau_{i}+\varepsilon_{i}^{-1} m \sinh \varepsilon_{i} \tau_{i} & \varepsilon_{i}^{-1} \sigma p_{i} \sinh \varepsilon_{i} \tau_{i} \\
\varepsilon_{i}^{-1} \sigma p_{i} \sinh \varepsilon_{i} \tau_{i} & \cosh \varepsilon_{i} \tau_{i}-\varepsilon_{i}^{-1} m \sinh \varepsilon_{i} \tau_{i}
\end{array}\right)
$$

That is exactly the form of the Green function for Dirac particle with $\delta\left(t-t^{\prime}\right)$-time dependence in the potential, where $t^{\prime}$ is the moment of $\delta$-kick. For $\kappa=0$, the first term gives the $\delta\left(p_{1}-p_{2}\right)$ dependence and the product of two $4 \times 4$-matreces in (75) gives one matrix of the form (35).

Let us find out the change of the form of the integral of motion for free Dirac particle related to the $\delta$-kick, for which $t^{\prime}=0$. Obviously, for negative times the form of the integrals of motion operators is the same as for free particle. But for positive times the form is changed. So, for the form of the invariant, which is initial momentum, one obtaines

$$
\left(\hat{p}_{0}(t)\right)_{p p^{\prime}}=\Theta(-t) p \delta\left(p-p^{\prime}\right)+\Theta(t) \int p^{\prime \prime}(\exp [i \kappa V])_{p p^{\prime \prime}}(\exp [i \kappa V])_{p^{\prime} p^{\prime \prime}}^{*} d p^{\prime \prime}
$$

where

$$
\begin{array}{ll}
\Theta(t)=1, & t>0 ; \\
\Theta(t)=0, & t<0 .
\end{array}
$$

The matrix $(\exp [-i \kappa V])_{p_{1} p_{2}}$ is proportional the unity matrix in four-space.

Thus, we have found the kernel of the time-dependent integral of motion for the kicked Dirac particle. This integral of motion plays the role of the initial momentum of the relativistic quantum motion. 


\section{Conclusion}

We have found some time-dependent integrals of motion for relativistic quantum particles. This consideration of time-dependent integrals of motion is motivated by the problem of pair creation in heavy ion collisions. Such processes may be modeled by fast time dependence of the interaction potential in Dirac equation. Due to this it is interesting to consider the constant of the motion in such type of models. The results of the paper are given in the explicit expressions (38)-(42) for the analog of the initial coordinate operator which is the integral of motion for the Dirac particle and in (76) for the analog of the initial momentum, which is the integral of motion for the kicked Dirac particle. We have found the time-dependent integral of motion for free Dirac particle given by (67), which is initial position operator by Newton and Wigner. Also the time-dependent integrals of motion related to initial spin and angular momentum observables (47) of free Dirac particle are the result of the analysis presented. It is worthy to study other time-dependent integrals of motion, which may be related to the high-energy processes modeled by interaction potentials dependent on time. One should note that in all cases of the relativistic quantum problems, for which the propagators are known explicitly, it is possible to find the explicit expressions for the kernels of time-dependent integrals of motion using the procedure analogous to the procedure applied for finding the initial momentum invariant of the kicked Dirac particle.

\section{Acknowledgments}

One of the authors (V.I.M.) would like to acknowledge Grupo de Física-Matemática, Complexo II, Universidade de Lisboa for kind hospitality and Russsian Foundation for Basic Research under 
the Project No. 96-02-17222 for partial support.

\section{References}

[1] A. Messiah, Mécanique Quantique, Nouveau Tirage (Dunop, Paris, 1964), Tome II.

[2] P. Ermakov, Univ. Izv. (Kiev) 20, N9, 1 (1880).

[3] H. R. Lewis, Phys. Rev. Lett., 18, 510; 636 (1967).

[4] H. R. Lewis and W. Riesenfeld, J. Math. Phys. 10, 1458 (1969).

[5] I. A. Malkin and V. I. Man'ko, Phys. Lett. A 32, 243 (1970).

[6] I. A. Malkin, V. I. Man'ko, and D. A. Trifonov, J. Math. Phys. 14, 576 (1973).

[7] I. A. Malkin, V. I. Man'ko, and D. A. Trifonov, Phys. Rev. D 2, 1371 (1970).

[8] V. V. Dodonov, I. A. Malkin, and V. I. Man'ko, J. Phys. A: Math. Gen. 8, L19 (1975).

[9] L. F. Urrutia and E. Hernandez, Intern. J. Theor. Phys. 23, 1105 (1984).

[10] O. Castaños and R. López-Peña, J. Phys. A: Math. Gen. 25, 6685 (1992).

[11] G. Profilo and G. Soliani, Ann. Phys. (New York) 229, 160 (1994).

[12] O. Castaños, R. López-Peña, and V. I. Man’ko, J. Phys. A: Math. Gen. 27, 1751 (1994).

[13] J. Cariñena, J. Fernández-Núñez, and E. Martínez, Lett. Math. Phys. 23, 51 (1991); Rep. Math. Phys. 31, 189 (1992).

[14] H. R. Lewis, Phys. Fluids B 2, 2551 (1990). 
[15] H. R. Lewis, P. G. L. Leach, S. Bouquet, and M. R. Feix, J. Math. Phys. 33, 591 (1992).

[16] H. R. Lewis and B. Abraham-Shrauner, Phys. Fluids B 4, 2024 (1992).

[17] H. R. Lewis, J. W. Bates, and J. M. Finn, Phys. Lett. A 215, 160 (1996).

[18] T. D. Newton and E. P. Wigner, Rev. Mod. Phys. 21, 400 (1949).

[19] S. S. Schweber, An Introduction to Relativistic Quantum Field Theory (Row, Peterson and Co., Evanston, Elmsford, N. Y., 1961).

[20] Z.K. Silagadze, "The Newton-Wigner position operator and the domain of validity of oneparticle relativistic theory," Preprint SLAC-PUB-5754 (1993).

[21] J. Schweppe et al., Phys. Rev. Lett. 51, 2261 (1983); 54, 1761 (1985).

[22] R. V. Mendes, Mod. Phys. Lett. 9, 2493 (1994).

[23] V. V. Dodonov and V. I. Man'ko, Invariants and Evolution of Nonstationary Quantum Systems, Proceedings of the Lebedev Physical Institute (Nova Science, Commack, N. Y., 1989), Vol. 183.

[24] I. A. Malkin and V. I. Man'ko, Dynamical Symmetries and Coherent States of Quantum Systems (Nauka, Moscow, 1979) [in Russian]. 